\title{
The DEMO Wall Load Challenge
}

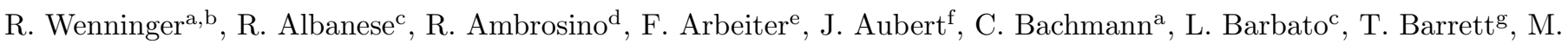

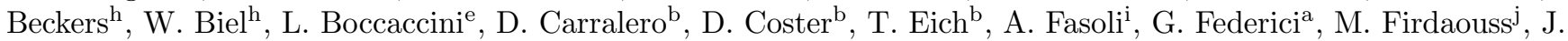
Graves $^{\mathrm{i}}$, J. Horacek ${ }^{\mathrm{k}}$, M. Kovari ${ }^{\mathrm{g}}$, S. Lanthaler ${ }^{\mathrm{i}}$, V. Loschiavo ${ }^{\mathrm{c}}$, C. Lowry ${ }^{\mathrm{l}}$, H. Lux ${ }^{\mathrm{g}}$, G. Maddaluno ${ }^{\mathrm{m}}$, F. Maviglia ${ }^{\mathrm{a}}$, R. Mitteau $^{\mathrm{q}}$, R. Neu ${ }^{\mathrm{b}}$, D. Pfefferle ${ }^{\mathrm{i}}$, K. Schmid ${ }^{\mathrm{b}}$, M. Siccinio ${ }^{\mathrm{b}}$, B. Sieglin ${ }^{\mathrm{b}}$, C. Silva ${ }^{\mathrm{q}}$, A. Snicker ${ }^{\mathrm{b}}$, F. Subba ${ }^{\mathrm{o}}$, J. Varje ${ }^{\mathrm{p}}$, H. Zohm $^{\mathrm{b}}$

\author{
${ }^{a}$ EUROfusion Programme Management Unit, Garching, Germany \\ ${ }^{b}$ Max-Planck-Institut für Plasmaphysik, Garching, Germany \\ ${ }^{c}$ Università di Napoli Federico II, Naples, Italy \\ ${ }^{d}$ Università di Napoli Parthenope, Naples, Italy \\ ${ }^{e}$ Karlsruhe Institute of Technology, Karlsruhe, Germany \\ ${ }^{f}$ CEA-Saclay, DEN/DM2S/SEMT, 91191 Gif-Sur-Yvette, France \\ ${ }^{g}$ Culham Centre for Fusion Energy, Culham Science Centre, Abingdon, UK \\ ${ }^{h}$ Institute of Energy- and Climate Research, Forschungszentrum Jülich GmbH; Germany \\ ${ }^{i}$ École Polytechnique Fédérale de Lausanne, Swiss Plasma Center, CH-1015 Lausanne, Switzerland \\ ${ }^{j}$ CEA, IRFM, F-13108 St Paul-Lez-Durance, France \\ ${ }^{k}$ Institute of Plasma Physics ASCR, Prague, Czech Republic \\ ${ }^{l}$ European Commission, B1049 Brussels, Belgium \\ ${ }^{m}$ ENEA Frascati, C.P. 65, 00044 Frascati, Rome, Italy \\ ${ }^{n}$ ITER Organization, St Paul Lez Durance, France \\ ${ }^{\circ}$ NEMO group, dipartimento di Energetica, Politecnico, I-10129 Torino, Italy \\ ${ }^{p}$ VTT Technical Research Centre of Finland, PO Box 1000, FI-02044 VTT, Finland \\ ${ }^{q}$ Instituto de Plasmas e Fusão Nuclear, Instituto Superior Técnico, Universidade de Lisboa, 1049 Lisboa,Portugal
}

\begin{abstract}
For several reasons the challenge, to keep the loads to the first wall within engineering limits, is substantially higher in DEMO compared to ITER. Therefore the pre-conceptional design development for DEMO ongoing now in Europe needs to be based on load estimates that are derived employing the most recent plasma edge physics knowledge.

An initial assessment of the static wall heat load limit in DEMO infers that the steady state peak heat flux limit on the majority of the DEMO first wall should not be assumed to be higher than $1.0 \mathrm{MW} / \mathrm{m}^{2}$. This compares to an average wall heat load of $0.29 \mathrm{MW} / \mathrm{m}^{2}$ for EU DEMO1 2015 assuming a perfect homogeneous distribution. The main part of this publication concentrates on the development of first DEMO estimates for charged particle heat loads, radiation heat loads, fast particle heat loads, disruption heat loads and particle loads including the resulting erosion rates. Employing an initial engineering wall design with clear optimization potential in combination with parameters for the flat-top phase (x-point configuration), loads up to $7 \mathrm{MW} / \mathrm{m}^{2}$ (penalty factor for tolerances etc. not applied) have been calculated. Assuming a fraction of power radiated from the x-point region between $1 / 5$ and $1 / 3$, peaks of the total power flux density due to radiation of $0.6-0.8 \mathrm{MW} / \mathrm{m}^{2}$ are found in the outer baffle region. Based on the first wall erosion limit and using a conservative assumption for the plasma parameters, the clearance between plasma and wall needs to be more than $0.3 \mathrm{~m}$.

This first review of wall loads and the associated limits in DEMO underlines clearly a significant challenge, that necessitates substantial engineering efforts as well as a considerable consolidation of the associated physics basis.
\end{abstract}

\section{Introduction}

The recent years of ITER design finalization have revealed that the plasma surface interaction requirements for integration of the first wall were previously underestimated. The DEMO design faces an even higher challenge. Compared to ITER the European DEMO design EU DEMO1 2015 [1] implies a fusion power that is four times higher and a major radius that is only 1.5 times higher. In addition, as the DEMO blanket has to perform efficient Tritium breeding and energy conversion, its first wall based on EUROFER-97 (ferritic-martensitic steel) instead of $\mathrm{Cu}$ as a heat conductor material is assessed to have a heat load limit that is significantly lower than extensive parts of ITER's first wall. Due to this, plasma surface interaction solutions for the first wall need to be integrated in the DEMO design from the pre-conceptual design phase, which is ongoing now. Moreover, various key design decisions for DEMO (e.g. double null configuration or high heat flux limiters at the first wall) are dependent on open questions on plasma surface interaction and SOL transport. 
This publication describes the status of knowledge with respect to the question, if the wall and the plasma in DEMO can be designed in a way, that the wall loads stay within acceptable limits. The main focus is on the prediction of wall loads based on simple considerations without entering too far related engineering considerations. Static loads are more featured compared to dynamic loads, which need to be investigated more in the future. It has to be stressed that this is a relatively early report, intended to create awareness of the gaps that need to be closed. The investigations presented in this paper are based on the design EU DEMO1 2015 [1], which includes a lower single-null magnetic configuration and an ITER-like divertor.

After the introduction, several technical first wall limits are introduced (section 2). After this, some basic information like the average static heat load distribution on the first wall of DEMO (subsection 3.1), relevant load types (subsection 3.2) and a conservative set of assumptions on the distribution of power to various key power loss channels (subsection 3.3) is presented. The bulk of the publication illustrates DEMO extrapolations for the following load types: Thermal charged particle heat loads (section 4 ), radiation heat loads (section 5), fast particle heat loads (section 6) disruption heat loads (section 7) and particle loads (section 8).

\section{Technical load limits for the first wall}

The baseline first wall designs assumed in this publication consist of a few $\mathrm{mm}$ of $\mathrm{W}$ amour joined onto a EUROFER-97 structure integrating numerous parallel cooling channels a few millimeters below its surface. The options for the coolant are pressurized $\mathrm{H}_{2} \mathrm{O}$ or He. In order to obtain a first indication of the static heat load limit of the first wall and its main dependencies calculations with the code RACLETTE [2] have been carried out (table 1). RACLETTE evaluates the thermal response of all components involved in the heat removal process. It includes all key heat transfer processes like evaporation, melting, radiation and water boiling and considers corresponding limits. Based on the loss of strength at high temperatures, the temperature limit of $550^{\circ} \mathrm{C}$ typically considered in the breeding blanket design was defined for EUROFER-97. Thermal stresses and failure by ratcheting is therefore not considered.

The EUROFER-97 temperature limit was found to be the driving criteria for the cases considered here. Apart from the coolant type and temperature $\vartheta_{\text {cool }}$ the minimum thickness $t$ of the EUROFER-97 structure between $\mathrm{W}$ and coolant has the most eminent impact on the first wall heat flux limit. Smaller first wall channels would allow further reduction of $t$ at the cost of increased pumping power requirements, higher fabrication cost, and higher manufac-
Table 1: Maximum heat load sustainable at the blanket before reaching the critical temperature in EUROFER-97 $\left(550^{\circ} \mathrm{C}\right)$, for different thicknesses $t$ of EUROFER-97 between coolant and W, different coolant temperatures $\vartheta_{\mathrm{cool}}$ and $\mathrm{H}_{2} \mathrm{O}$ cooling respectively He cooling

\begin{tabular}{|l|l|l|l|}
\hline \multicolumn{4}{|c|}{$\mathrm{H}_{2} \mathrm{O}$ cooling } \\
\hline & $\vartheta_{\text {cool }}=275^{\circ} \mathrm{C}$ & $\vartheta_{\text {cool }}=315^{\circ} \mathrm{C}$ & \\
\hline$t=2 \mathrm{~mm}$ & $2.00 \mathrm{MW} / \mathrm{m}^{2}$ & $1.73 \mathrm{MW} / \mathrm{m}^{2}$ & \\
\hline$t=3 \mathrm{~mm}$ & $1.70 \mathrm{MW} / \mathrm{m}^{2}$ & $1.47 \mathrm{MW} / \mathrm{m}^{2}$ & \\
\hline \multicolumn{4}{|c|}{$\mathrm{He}$ cooling } \\
\hline & $\vartheta_{\text {cool }}=330^{\circ} \mathrm{C}$ & $\vartheta_{\text {cool }}=380^{\circ} \mathrm{C}$ & $\vartheta_{\text {cool }}=430^{\circ} \mathrm{C}$ \\
\hline$t=2 \mathrm{~mm}$ & $0.70 \mathrm{MW} / \mathrm{m}^{2}$ & $0.54 \mathrm{MW} / \mathrm{m}^{2}$ & $0.38 \mathrm{MW} / \mathrm{m}^{2}$ \\
\hline$t=3 \mathrm{~mm}$ & $0.67 \mathrm{MW} / \mathrm{m}^{2}$ & $0.52 \mathrm{MW} / \mathrm{m}^{2}$ & $0.37 \mathrm{MW} / \mathrm{m}^{2}$ \\
\hline
\end{tabular}

turing precision requirements.

We currently assume that the steady state peak heat flux limit on the majority of the DEMO first wall is not higher than $1 . M W / m^{2}$. This is much lower than the value being considered on the first wall of ITER, which currently assume in the areas mostly loaded $4.7 \mathrm{MW} / \mathrm{m}^{2}[3]$. The lower power handling capability of the wall in DEMO arises from [4]: (i) the requirement to breed tritium that imposes thin and low n-absorbing PFCs, (ii) the higher coolant temperature for efficient power conversion, (3) the need to use materials able to withstand high neutron fluency and significant radiation damage and with low activation. In the case of DEMO it is necessary to use EUROFER-97 as heat sink material rather than $\mathrm{CuCrZr}$ [5] as in the case of ITER, noting that the latter has roughly ten times higher thermal conductivity.

In addition to these designs, first concepts of alternative designs with higher heat flux capabilities are currently under discussion. These include design concepts with

- lower $\vartheta_{\text {cool }}$, for which an integration in the primary heat transfer system might not be possible (Could reduce the net electric power output of the plant.)

- different material choices like the usage of $\mathrm{Cu}$-alloys as heat sink (Necessitates more frequent exchange of components.)

- more complex first wall options (e.g. including HyperVapotrons) (Could increase significantly the total plant costs.)

An extreme option would be local wall components with high heat flux capabilities $\left(\approx 20 M W / m^{2}\right.$ for a limited duration) based on the ITER monoblock technology [6].

Another critical question is, if the dynamic loads exposed to the first wall of DEMO are leading to any limit of the first wall component being exceeded. For dynamic events, where the heat deposition is faster than the heat removal, the surface melting limit of $\mathrm{W}\left(\approx 3400^{\circ} \mathrm{C}\right)$ could be come critical. Expressed in terms of the heat impact factor, which accounts for the heat diffusion process, melting of $\mathrm{W}$ surfaces starts from around $50 \mathrm{MJ} /\left(\mathrm{m}^{2} \sqrt{s}\right)$ [7]. During dynamical events also the temperature margin of the EUROFER-97 structure might be quickly exceed. It is expected that this limit is more constraining than 
the W melt limit. More information on this can be found in [8]. Furthermore, it is currently not completely obvious, how constraining $\mathrm{W}$ recrystallization $\left(\approx 1200^{\circ} \mathrm{C}\right)$ - especially in the case of periodic excursions of this temperature - will be.

Also particle loads at the first wall of DEMO are a concern. As the W amour layer has a thickness of only a few $\mathrm{mm}$, the question is raised, if static or periodic dynamic particle loads can lead to a total erosion that is of the order of this thickness. Furthermore, the eroded W dust can be deposited in areas, from which it could fall into the plasma and hence increase the disruptivity of the tokamak.

\section{Basic information}

\subsection{Average heat load}

To obtain a first very crude assessment of wall heat load in DEMO, the total charged particle heating power (alpha heating power and auxiliary heating power) of $457 \mathrm{MW}$ is divided by the estimated wall surface of $1556 \mathrm{~m}^{2}$ obtaining an average heat load of $0.29 \mathrm{MW} / \mathrm{m}^{2}$. Hence, in case of a limit of $1 M W / m^{2}$ a total peaking factor of up to 3.4 would be acceptable. To obtain a more detailed picture, predictions for each relevant load type have to be carried out.

For comparison, in the ITER case with the highest total charged particle heating power the average heat load is $153 \mathrm{MW} / 800 \mathrm{~m}^{2}=0.19 \mathrm{MW} / \mathrm{m}^{2}$. The load on the first wall is specified up to $4.7 \mathrm{MW} / \mathrm{m}^{2}$ corresponding to a peaking factor of 25 .

\subsection{Relevant load types}

A first step towards a more detailed load assessment is to identify a preliminary list of the relevant load types:

- Stationary heat loads

- Thermal charged particles including blob effects

- Radiation

- Neutrals

- Fast particles

- Dynamic heat loads

- Limiter configuration during ramp-up/down

- ELM filaments

- Confinement transients (e.g. H-L-transition)

- Vertical displacement events / disruptions

- Particle Loads

- Steady state and dynamic first wall erosion yield
Certainly, some of these load types are more dominant than others. However, it is essential to obtain estimates for all of them.

\subsection{Assumptions on the power distribu- tion}

For the investigation of stationary heat loads several assumptions on the power distribution in the plasma have to be made. The power crossing the separatrix $P_{\text {sep }}$ of $154 \mathrm{MW}$ corresponds to 1.16 times the L-H-threshold power $P_{L H}$ calculated employing the scaling from Martin [9]. ${ }^{1}$ It is assumed that the maximum value of $P_{s e p}$ is 1.5 times the nominal value of $P_{\text {sep }}$. This does not include the case of an unforeseen H-L-transition during the flattop phase of the pulse, which needs to be investigated separately. A simple model is assumed, in which $P_{s e p}$ is distributed into three channels: (1) a part associated with the standard SOL heat transport via charged particles as described in the Goldston model [11] with $\lambda_{q}=1 \mathrm{~mm}$ corresponding roughly to the scaling described in $[12,13],(2)$ a part associated with the charged particle blob transport, where we assume a much higher e-folding length of $100 \mathrm{~mm}$ as justified in subsection 4.1 and (3) a part that is going into radiation in the SOL and divertor. Only a negligible fraction of power in channel (1) arrives at the first wall and channels (2) and (3) correspond to the main charged particle loads of the first wall.

There are significant uncertainties in the distribution of $P_{\text {sep }}$ to these channels. Table 2 shows two power distributions, which are conservative with respect to first wall loads in channel (2) respectively (3). As discussed in subsection 4.1, the maximum power that could be transported by blobs is assumed to be $0.2 P_{\text {sep }}$. Aslo it is assumed that in a high radiation event it is assumed that up to $100 \%$ of $P_{\text {sep }}$ can be radiated.

Table 2: Conceivable limit power distributions, in which the power deposited onto the wall by charged particles respectively SOL / Divertor radiation is maximized

vertor radiation is maximized
\begin{tabular}{|l|l|l|l|}
\hline $\begin{array}{l}\text { Maximized } \\
\text { channel }\end{array}$ & Charged particle & $\begin{array}{l}\text { SOL / Divertor } \\
\text { radiation }\end{array}$ \\
\hline $\begin{array}{l}\text { Fraction } \\
\text { into } \\
\lambda_{q}=1 \mathrm{~mm}\end{array}$ & $\%$ & 40 & 0 \\
\hline $\begin{array}{l}\text { Fraction } \\
\text { into } \lambda_{q}= \\
100 \mathrm{~mm}\end{array}$ & $\%$ & 20 & 0 \\
\hline $\begin{array}{l}\text { Fraction } \\
\text { into SOL / } \\
\begin{array}{l}\text { Divertor } \\
\text { radiation }\end{array}\end{array}$ & $\%$ & 40 & 100 \\
\hline
\end{tabular}

\footnotetext{
${ }^{1}$ The Martin scaling [9] is based on data from $\mathrm{C}$ wall devices. A reduction of $P_{L H}$ by $20-30 \%$ has been observed in $\mathrm{W}$ wall devices [10]. Also, for the ITER design point the 95\%-confidence interval expands from $\approx 50 \%$ to $\approx 200 \%$ of the scaled value. Finally a reasonable margin $P_{L H}$ should be allowed to ensure sufficient controllability and confinement quality
} 


\section{Charged particle heat loads}

\subsection{The role of blobs}

Blobs are coherent structures of denser plasma compared to the SOL background plasma elongated in the parallel direction. It has been predicted that there are two regimes for the perpendicular velocity $v_{\perp, b l o b}$ of the blobs [14]: At low SOL resistivity, in the sheath limited regime, $v_{\perp, b l o b} \propto$ $1 / \delta_{b}^{2}$, where $\delta_{b}$ is the blob size. At high SOL resistivity, in the inertial regime, $v_{\perp, b l o b} \propto \sqrt{\delta}_{b}$. The transition between the two regimes has been shown experimentally [15] to be determined by the collisionality at the divertor plate. The inertial regime is especially reached, if the condition

$$
\Lambda:=\frac{L_{\|} / c_{s, \text { Div }}}{1 / \nu_{e, i, \text { Div }}} \frac{m_{e}}{m_{i}}>1
$$

is fulfilled, where $L_{\|}$is the connection length, $c_{s, D i v}$ is the sound speed in the divertor and $\nu_{e, i, D i v}$ is the electronion collision frequency. While $\Lambda>1$ is not easily achieved in recent devices, it has been shown that for realistic DEMO parameters it will be fulfilled and hence DEMO will be in the inertial regime [16]. Consequently, the particle transport associated with the blobs is much more pronounced. On the contrary, the influence on the heat transport - which cannot be directly inferred from the particle transport - is at the moment not fully understood.

It has been observed that up to $50 \%$ of the particles transport can be facilitated by blobs [17]. We assume a simple model, in which $50 \%$ of the associated power is transferred by the electrons to the divertor and $50 \%$ (25\% of the nonradiated part of $P_{\text {sep }}$ ) is represented in ions propagates with the blob to wall. This compares to [18] where the difference between heating power and the sum of power to the target and radiated power has been found to be about $40 \%$ in ASDEX Upgrade at highest densities. This difference, that is exposed to extensive measurement uncertainties, could be interpreted as the power that is deposited at the wall. Having in mind these values we have associated up to $20 \%$ of $P_{\text {sep }}$ to the long- $\lambda_{q}$-channel in table 2 .

In [16] first estimates for the power flux densities to the wall due to blobs are presented. Field line tracing in 2D has been applied adding the velocity component associated with the perpendicular motion of the blobs. Here the pessimistic assumption has been used, that the parallel elongation of the blobs is negligible at birth. ${ }^{2}$ The free parameters, which also represent urgent experimental investigation needs, are:

- the fraction of power transferred by the blobs $f_{p}$

- the size of the blobs $\delta_{b}$

- the velocity scaling factor $f_{v}{ }^{3}$

\footnotetext{
${ }^{2}$ An parallely elongated blob would spread the power on a significantly larger area of the first wall.

${ }^{3}$ This is a correction factor accounting for the fact that the employed equation for the perpendicular blob velocity is describing an upper limit.
}

Assuming $P_{\text {sep }, \text { tot }}=231 M W$ and making reasonable assumptions on the blobs $\left(f_{p}=0.2, \delta_{b}=12 \mathrm{~cm}, f_{v}=0.5\right)$ leads to wall heat loads due to blobs of $0.16 \mathrm{MW} / \mathrm{m}^{2}$. Going to a more conservative but still conceivable set of parameters $\left(f_{p}=0.3, \delta_{b}=15 \mathrm{~cm}, f_{v}=0.7\right)$ gives $0.49 \mathrm{MW} / \mathrm{m}^{2}$ at the wall. It has to be stressed that the transfer from $2 \mathrm{D}$ wall load calculations with an idealized wall to $3 \mathrm{D}$ calculations with an engineering design of the wall can imply a significant increase of the resulting heat loads.

In the $3 \mathrm{D}$ wall load calculations presented in subsection 4.3 exclusively blob transport with a corresponding e-folding length $\lambda_{q, b l o b}$ is considered. $\lambda_{q, b l o b}$ is estimated assuming local heat conservation $\left(\nabla_{\|} q_{\|}=-\nabla_{\perp} q_{\perp}\right)$ and approximate differential operators $\left(\nabla_{\|} \approx 1 / L_{\|}, \nabla_{\perp} \approx 1 / \lambda_{q, b l o b}\right)$. For the heat fluxes we use $q_{\|}=-\chi_{0} T^{5 / 2} d T / d s$ and $q_{\perp}=3 e n_{u p} T f_{i n t} v_{\perp}$, where for $v_{\perp}$ we use the scaling for the inertial regime and $f_{\text {int }}$ is a factor that accounts for the intermittent nature of the blob transport. In [19] it is reported, that in ASDEX Upgrade L-mode plasmas typically $\approx 2 \%$ of a time trace from lithium beam emission spectroscopy consists of blobs $(\geq 2.5 \sigma)$. To be conservative we use $f_{\text {int }}=0.04$ in combination with $n_{u p}=3 \times 10^{19} \mathrm{~m}^{-3}$, $L_{\|}=150 \mathrm{~m}, T_{\text {sep }}=200 \mathrm{eV}$ and a blob size of $10 \mathrm{~cm}$, which gives $\lambda_{q, b l o b} \approx 100 \mathrm{~mm}$.

\subsection{The role of ELMs}

In the ITER case the majority of the anticipated base load limit of $4.6 \mathrm{MW} / \mathrm{m}^{2}$ on the top panels of the first wall is associated with ELMs [20]. A first review of the divertor temperature limit during ELMs in EU DEMO1 2015 came to the conclusion that an ELM mitigation method is required, that reduces the relative ELM size by a factor of 15 to 90 [1]. Consequently, various ELM mitigation methods are discussed [21] and first feasibility assessments have been started. However it is unclear, which method will be selected and which are the characteristics of any remaining plasma edge perturbations, the associated filamentary actives and the resulting wall loads. Depending on the reliability of the ELM mitigation method plasma and wall might have to be designed to also sustain phases with unmitigated type-I ELMs.

\subsection{Static charged particle wall loads in the flattop phase}

The ITER experience has shown that 3D investigations are required to predict the peak heat loads due to plasma wall interaction with a wall that has typical engineering features (e.g. gaps and chamfers). A first set of such investigations has been carried out based on an initial engineering design of the first wall in DEMO. It should be noted that this wall design is initial and not optimized and even exhibits some substantial weaknesses, such that significant modifications will be required. Hence the calculated peak heat fluxes on this design can only provide 
a first indication and not an information on the loads on the final design.

The code PFCFlux [22] has been employed for the power flux density calculations. According to subsection 3.3 a power crossing the separatrix of $46 \mathrm{MW}$, which is distributed with $\lambda_{q}=100 \mathrm{~mm}$, has been assumed. PFCFlux calculates the heatflux mapping from the outer midplane to the wall and the associated shadowing using 3D fieldline tracing.

Figure 1 shows the peak heat flux density per blanket module. The peak heat loads of $6-7 M W / m^{2}$ are observed at the inner and outer baffle region (blanket-divertor transition region). Considering the relatively extensive angle between flux surfaces and first wall in this area, there is obviously some optimization potential. However, it is important to recall that in the ITER case penalty factors of in total up to 2.44 accounting for various deviations (appearing during, design, manufacturing, assembly and operation) from the idealized situation assumed have been introduced [20]. Also it should be stressed that especially the out baffle is also highly loaded by radiation (section $5)$. As there is not much to be done about the radiation load the charged particle heat load to this area has to be reduced by almost two orders of magnitude. Also at the top of the main chamber relatively high loads of up to $\approx 1.0 \mathrm{MW} / \mathrm{m}^{2}$ are observed. Besides the necessity to also reduce these loads, the load evolution during upward vertical displacement events have to be thoroughly investigated.

Figure 2 shows as an example the power flux density distribution on the inner baffle (module 1). The edges in the toroidal direction have a radius of $100 \mathrm{~mm}$. The peak heat loads appear in this area just before shadowing from other components sets in. This early version of the mesh does not include a radius or a chamfer or any side face in the poloidal direction, which is relevant due to the poloidal gap between the blanket elements. The inclusion of this feature could lead to a further increase in the power flux density.

\subsection{Static charged particle wall loads in limited configurations in ramp-up and ramp-down}

The first wall loads in the diverted configuration can in principle be managed by increasing the wall clearance ${ }^{4}$ up to a point, at which the impact on the vertical stability [23] and hence on overall device performance [24] becomes intolerable. In contrast, in a limited configuration (e.g. during ramp-up or ramp-down), there is no free parameter like the wall clearance in a diverted configuration. However, there is the option to diverge from the baseline design option (i.e. wall contact at a low heat flux wall

\footnotetext{
${ }^{4}$ In the baffle region this might necessitate a modification of the divertor design.
}

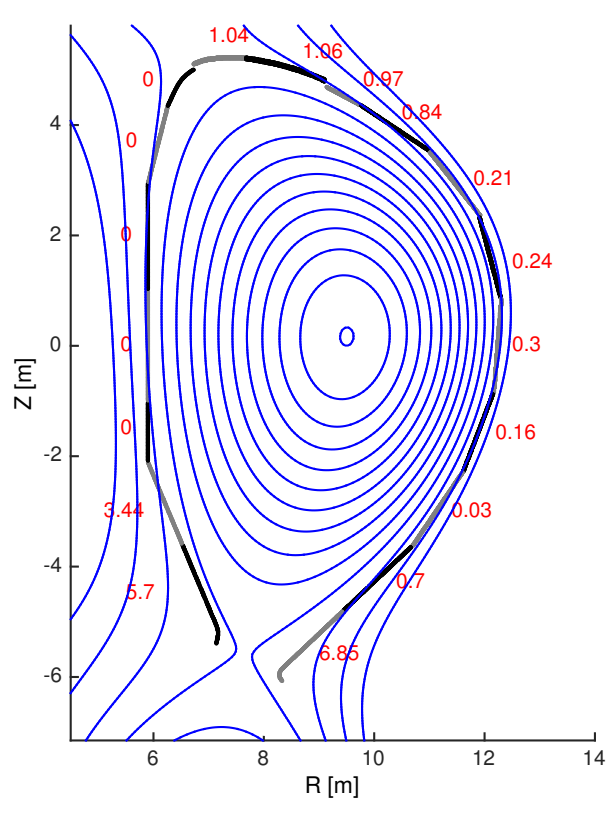

Figure 1: Peak power flux density for all 18 blanket modules calculated with PFCFlux assuming 46MW crossing the separatrix to be distributed with $\lambda_{q, b l o b}=100 \mathrm{~mm}$ : The front faces of the blanket models are alternately shown in black and gray. Flux surfaces are shown in blue.

component) and include one or more dedicated high heat flux limiters into the design, where the wall contact happens.

Planned limiter configurations have usually wall contact at the inboard or outboard side and both of these options have advantages and disadvantages. An inboard limited configuration would have the advantage, that the electrical field during breakdown can be higher and consequently a higher pump-down target pressure and a lower pump-down duration ${ }^{5}$ could be possible with or without EC breakdown assistance.

Another difference between inboard and outboard limited plasmas is related to the e-folding length $\lambda_{q}$. For inboard limited plasmas a near SOL and a main SOL component are expected. Using an inboard limited configuration with $5 \mathrm{MA}$ we estimate $\lambda_{q, \text { main }}^{\text {OMP }} \approx 45 \mathrm{~mm}$ (based on scalings from [25]) and $\lambda_{q, \text { near }}^{O M P} \approx 2.0 \mathrm{~mm}$ (based on the Goldston model [11]). A typical DEMO equilibrium has a flux expansion between OMP and IMP of 1.7 leading to $\lambda_{q, \text { main }}^{I M P} \approx 76 \mathrm{~mm}$ and $\lambda_{q, \text { near }}^{I M P} \approx 3.4 \mathrm{~mm}$. For the distribution of the power between the two components a large variety observed on various devices is reported in [26], concluding that it can be expected hat the parameter

\footnotetext{
${ }^{5}$ Based on current estimates for the first wall temperature during plasma operation and dwell time, hydrogen transport simulations assuming a neutron damaged wall suggest that strong outgassing from these neutron generated defects in $\mathrm{W}$ will increase the pump down time to more than $1000 \mathrm{sec}$ to reach a base pressure of $5 \times$ $10^{-4} \mathrm{~Pa}$.
} 


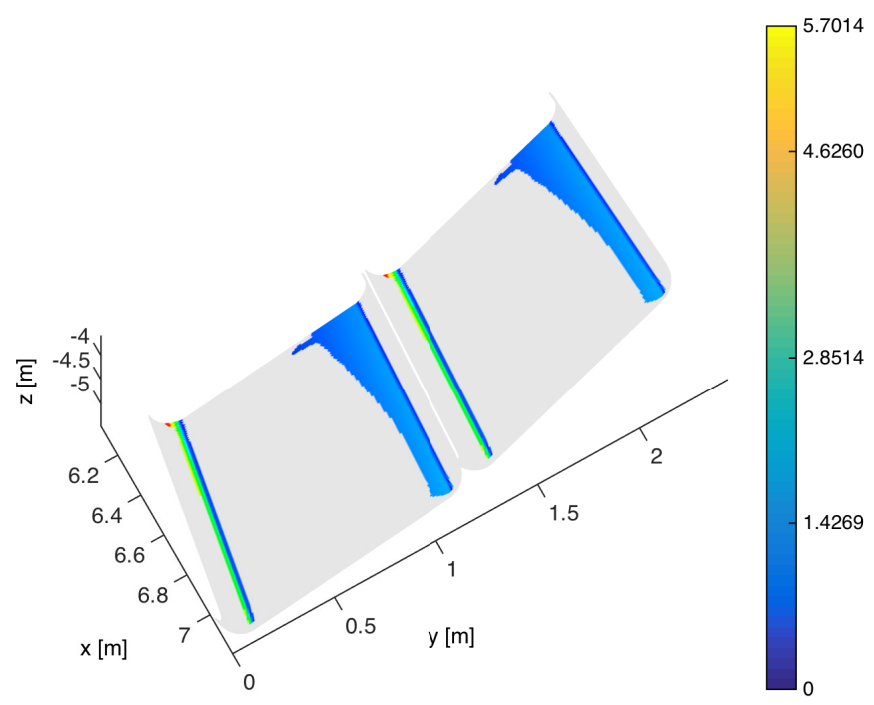

Figure 2: Power flux density distribution (in $M W / m^{2}$ ) on the inner baffle (module 1) in a view from the top: The grey areas are shadowed.

$R_{q}=q_{\| 0, \text { near }} / q_{\| 0, \text { main }}$ falls in the range between 1 and 6. We suggest to adopt the same approach for DEMO until more understanding of the determination of $R_{q}$ is obtained.

For outboard limited plasmas the knowledge base is much weaker, which - considering that this is an option for DEMO - should be changed. We use the finding, that in JET $\lambda_{q}$ for outboard limited plasmas can be up to 7.5 times lower than $\lambda_{q, \text { main }}$ for inboard limited plasmas [27]. This leads to a conservative estimate for $\lambda_{q}^{O M P}$ for outboard limited plasmas in DEMO of $6 \mathrm{~mm}$.

The most preferable option for limiter positioning in DEMO in terms of maintainability is to put them inside ports. This would allow, that the limiter could be exchanged several times during the life time of DEMO. Especially the ports at the outer midplane, which are more poloidally than toroidally elongated, seem to be very relevant.

The maximum power crossing the separatrix as usual is an important parameter for the calculation of the wall loads. Similar to ITER it is assumed that the limited to $\mathrm{x}$-point transition happens at about 5MA. Up to this point the ohmic power launched into the plasma has been calculated to be less than $3 \mathrm{MW}$. It is assumed that despite of the breakdown phase ${ }^{6}$ no auxiliary power is launched to the plasma during the phase with limited configuration and hence $P_{\text {sep }}=3 M W$ is used. We note that for ITER the rule $P_{\text {sep }}[M W]=I_{P}[M A]$ has been used. Due to the negative $d W_{\text {plasma }} / d t$ the power crossing the separatrix

\footnotetext{
${ }^{6}$ Here EC powers of up to $6 \mathrm{MW}$ are currently considered.
}

could be higher during the limiter phase at the end of the ramp-down, however to assess this the investigation of the DEMO ramp-down needs to progress. If the plasma is perturbed into a limited configuration during the flattop phase, $P_{\text {sep }}$ can be higher by orders of magnitude.

\section{Radiation heat loads}

In order to be able to manage the power exhaust, DEMO has to operate with significantly higher radiation fractions than ITER. The optimum impurity mix to achieve simultaneously (1) divertor protection, (2) H-mode operation and (3) optimized fusion performance has been investigated $[23,16]$, but still needs further substantiation. Until now it seems to be clear that because of the relatively low fuel dilution higher $\mathrm{Z}$ impurities like $\mathrm{Ar}$ or $\mathrm{Kr}$ are interesting candidate seeding species. A second seeding species with lower $\mathrm{Z}$ radiating more efficiently in the divertor might be added. It needs to be granted that the peak wall loads due to radiation in combination with other loads do not exceed the wall load limits.

In a first approach for the estimation of the static radiation wall loads in DEMO it has been assumed that the radiation source density is constant on flux surfaces. Figure 3 shows the total radiation load on the first wall for EU DEMO1 2015 (303MW radiated from inside and 154MW from outside the separatrix $)^{7}$. This is based on the assumption, that the radiation source density is constant on flux surfaces. A peak load of $\approx 0.4 M W / m^{2}$ is observed at the outer mid plane.

However, especially in plasmas with a (partially) detached outer divertor significant levels of radiation peaking in the x-point region have been observed [31]. Figure 4 shows the situation with a radiation source of $150 \mathrm{MW}$ concentrated in the X-point. A peak load of $\approx 0.8 \mathrm{MW} / \mathrm{m}^{2}$ is observed on the divertor dome, which can be designed as a high heat flux component. In the outer baffle region up to $0.5 \mathrm{MW} / \mathrm{m}^{2}$ are calculated. Extrapolating this to the theoretical worst case limit where all heating power $P_{\alpha}+P_{a u x}=457 M W$ is radiated from a highly localized source at the $\mathrm{X}$-point leads to $\approx 1.5 \mathrm{MW} / \mathrm{m}^{2}$. These loads in combination with the loads from other load types - especially thermal charged particle loads, which have a tendency to peak also in the baffle region - need to be compared with the limits described in section 2 .

Unlike $\mathrm{C}, \mathrm{N}$ and Ne that only strongly radiate at temperatures expected in the divertor/SOL $(<100 \mathrm{eV})$, higher $\mathrm{Z}$ impurities like $\mathrm{Ar}, \mathrm{Kr}$ or $\mathrm{Xe}$ also radiate significantly at temperatures further inside the separatrix. Therefore, we can assume that in DEMO already a significant fraction of the heating power has been radiated before it can be conducted/convected across the separatrix. Furthermore, the

\footnotetext{
${ }^{7}$ The poloidal radiation load distribution is different than in [23], as a weakness of the analysis code has been identified and corrected recently.
} 


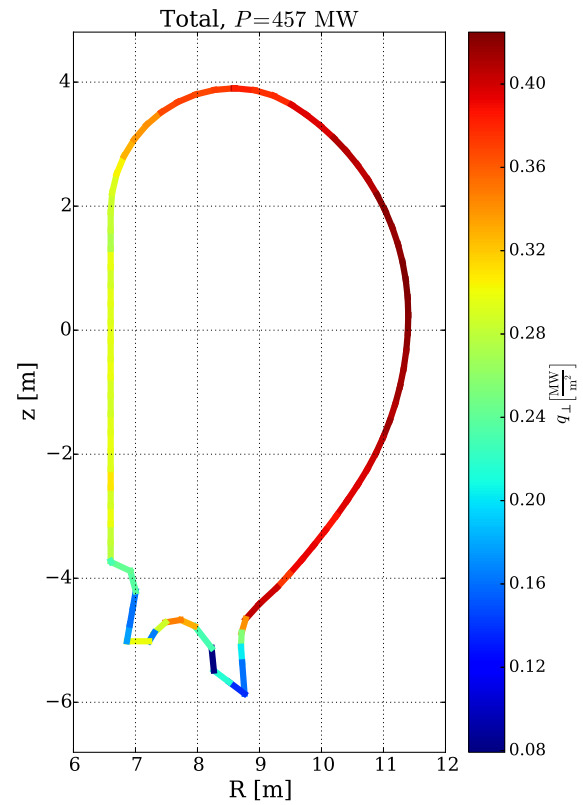

Figure 3: Total radiation load on the first wall of DEMO for a plasma with with $0.74 \% \mathrm{Ar}$ and $0.013 \% \mathrm{Xe}$ and $P_{\text {rad,tot }}=P_{\alpha}+P_{\text {aux }}=$ $457 \mathrm{MW}$, based on the assumption of a constant radiation source density on flux surfaces: The radial radiation source profiles have been obtained DEMO simulations with STRAHL [28] coupled to ASTRA [29, 30]

radiating volume in the core plasma in DEMO will be significantly larger than the radiating volume in the $\mathrm{x}$-point and divertor region. Both effects suggest that the scenario described above, where all heating power is radiated in the $\mathrm{x}$-point vicinity, is highly unlikely. Following experimentally observed ratios of divertor/x-point radiation to main chamber radiation as described in [32] for $\mathrm{N}, \mathrm{Ne}$ and $\mathrm{Ar}$ seeding on JET a scenario where maximally $150 \mathrm{MW}$ is radiated from the $\mathrm{x}$-point region seems more realistic, but still very conservative upper limit for a stable operating scenario. In fact considering a ratio of $1 / 5$ of divertor and $\mathrm{x}$-point to main chamber radiation for $\mathrm{Kr}$ seeding in $\mathrm{JET}$ as reported in [33], would suggest an upper limit for the $\mathrm{x}$-point radiator to be $60 \mathrm{MW}$.

Table 3 estimates the total radiation power load at the outer baffle for the two x-point radiation fractions $33 \%$ and $20 \%$. It is assumed that after subtracting the $\mathrm{x}$-point radiation power $P_{x-\text { rad }}$, the residual radiation power $P_{\text {res-rad }}$ has a radial/poloidal source distribution as assumed for figure 3 (i.e. radiation source density constant on flux surfaces in confined and SOL plasma). The peak of the total radiation power flux density on the first wall (not divertor or dome) for these two cases is found at the outer baffle and have values of $0.62 \mathrm{MW} / \mathrm{m}^{2}$ and $0.77 \mathrm{MW} / \mathrm{m}^{2}$.

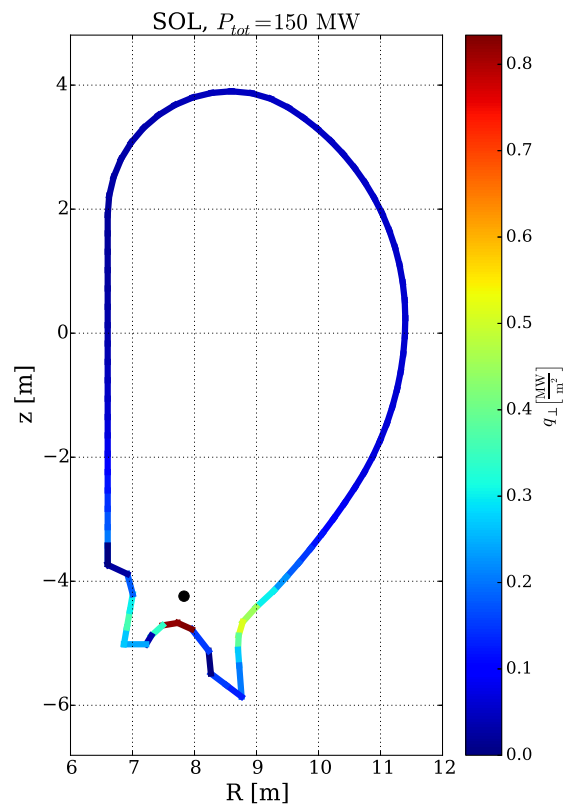

Figure 4: Radiation load on the first wall of DEMO based on the assumption that $P_{\text {sep }}=150 \mathrm{MW}$ is radiated from the x-point

Table 3: Total radiation power density (and breakdown) at the outer baffle for two distributions into $\mathrm{x}$-point radiation and residual radiation (i.e. radiation source density constant on flux surfaces in confined and SOL plasma)

\begin{tabular}{|l|l|l|}
\hline Fraction of x-point radiation & 0.33 & 0.20 \\
\hline$P_{x-\text { rad }}[\mathrm{MW}]$ & 151 & 91 \\
\hline$q_{\max , \text { bo }, x-\text { rad }}\left[\mathrm{MW} / \mathrm{m}^{2}\right]$ & 0.50 & 0.30 \\
\hline$P_{\text {res }- \text { rad }}[\mathrm{MW}]$ & 306 & 366 \\
\hline$q_{\max , \text { bo,res-rad }}\left[\mathrm{MW} / \mathrm{m}^{2}\right]$ & 0.27 & 0.32 \\
\hline$q_{\max , \text { oo }, \text { tot }}\left[\mathrm{MW} / \mathrm{m}^{2}\right]$ & 0.77 & 0.62 \\
\hline
\end{tabular}

\section{Fast particle heat loads}

First wall power loads by fast alpha particles were calculated using the well-established ASCOT code [34]. Losses that may be due to fluctuations and waves in the plasma have not been considered so far. The simulation was carried out for four different approximation of the background magnetic field: an axisymmetric 2D field (2D), a field including TF ripple due to finite number of TF coils (TF only), a field including TF ripple and ferritic inserts with full mass (TF FI full) and with half mass (TF FI half) to minimize the costs of the installation. The simulations were carried out until the alpha particles slowed down to local thermal energy, or until the alphas hit the first wall contour. As a first wall, a fully 3D CAD design was used enabling to detect possible hot spots or vulnerable components. Table 4 shows the global values for alpha particle losses. Two things are particularly interesting in this table. First, the mitigation of heat loads is very well obtained only with half of the mass in ferritic inserts. Secondly, the power loss and alpha losses does not correlate very well, 


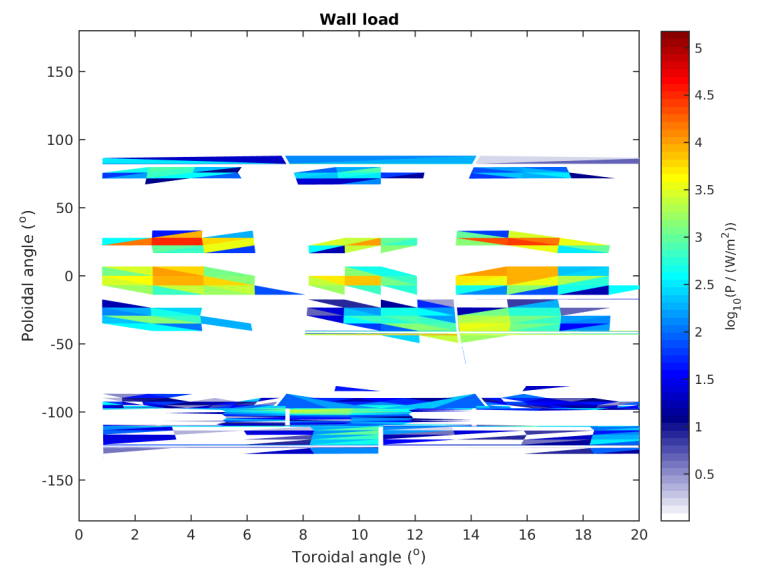

Figure 5: Alpha particle heat loads on the 3D blanket design of the DEMO wall using the 18-fold symmetry of the physical model with the additional red dots showing the positions of the wall hitting alphas.

i.e. the ferritic inserts tend to shield more particles with higher energies leading to lower lost powers while the absolute number of lost alphas is not dramatically different. Overall, the values obtained in this study are rather low. However, as shown in figure 5, this load is not to be distributed evenly along the wall, but rather peaked in both toroidal and poloidal directions. Even taking this into account, the maximum heat loads are well bellow the level of $0.1 \mathrm{MW} / \mathrm{m}^{2}$.

Table 4: Global loss parameters for four different configurations

\begin{tabular}{|l|l|l|l|}
\hline Configuration & $\begin{array}{l}\text { Alpha loss } \\
(\%)\end{array}$ & $\begin{array}{l}\text { Power loss } \\
(\%)\end{array}$ & $\begin{array}{l}\text { Lost power } \\
(\mathrm{kW})\end{array}$ \\
\hline 2D & 5.4 & 0.026 & 110 \\
\hline TF only & 7.7 & 0.15 & 640 \\
\hline TF FI full & 6.0 & 0.037 & 160 \\
\hline TF FI half & 6.6 & 0.052 & 220 \\
\hline
\end{tabular}

While this analysis revealed several interesting and important aspects, unfortunately, there has been several shortcomings including most importantly inaccuracy of the magnetic field, calculated by FEM-based method, leading to crossing of the magnetic field lines deep inside the plasma. Moreover, the size of the wall elements used in this study clearly is not fully optimized. For many elements the wall loads are under-/overestimated as the elements are either very small (only one high energy particle can hit it, overestimating the heat load) or too large (part of the element can be empty and the other part can have several recordings of alphas been hit, thus, underestimating the heat load). These shortcomings are being issued at the moment and the results will be published in more detail separately. However, we do not expect the main results, like the data shown in table 4 , to be quantitatively different even when the shortcomings have been relaxed. In a complementary study [35] the question of the relevance of the plasma response for the fast particle losses

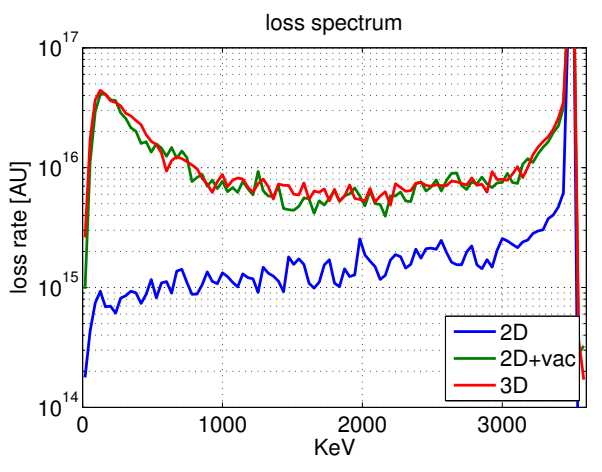

Figure 6: Showing loss rates as a function of energy for alpha particle distributions calculated assuming a $2 \mathrm{D}$ equilibrium without ripple (blue), a 2D +ripple model (green), a 3D equilibrium model (red)

has been investigated. This is motivated by the recent discovery [36], that the plasma response model employed to investigate the effect of axisymmetry breaking due to $\mathrm{n}=3$ resonant magnetic perturbation (RMP) coils has a very strong effect on fast ion confinement and losses. Two opposing approaches are compared, one where the symmetry breaking field calculated in absence of the plasma is added to an axisymmetric MHD equilibrium calculation (henceforth called the "2D +ripple" approach), while the other where a full 3D free boundary MHD equilibrium calculation naturally includes the plasma response within the 3D deformation of its flux-surfaces (henceforth called the "3D equilibrium" approach). Analyzing the fast particle trajectories for the two descriptions of the magnetic field for DEMO in the orbit code VENUS-LEVIS [37], it was found that the guiding center approximation was adequate for the study, essentially because the scale length of the magnetic field variation is much larger that the Larmor radius of $3.5 \mathrm{MeV}$ alpha particles. In addition, unlike in the $\mathrm{n}=3$ RMP study investigated previously [36], the magnetic ripple in DEMO (which has mode number $n=18$ ) does not cause a significant plasma response. Hence the standard "2D+ripple" approach and "3D equilibrium" approach reveal essentially the same plasma confinement properties and losses. This is indicated in Figure 6, which shows loss rates of alpha particle distributions plotted as a function of energy assuming a 2D equilibrium without ripple (blue), a 2D+ripple model (green), and a 3D equilibrium model (red). The figure also shows, that the ripple induced transport (diffusive-like losses) is strongest between $100-200 \mathrm{KeV}$, hence it could be a useful ash-removal mechanism.

\section{Disruption heat loads}

The damage of plasma facing components due to huge heat loads during disruptions is of great concern for DEMO. In the following a first estimate of the peak power flux density and the heat impact factor during unmitigated 
and mitigated disruptions in DEMO is presented. This is based on a plasma in EU DEM01 2015 with $I_{P}=19.6 \mathrm{MA}$, $\beta_{\text {pol,tot }}=1.0$ and a pre-disruption thermal energy of $0.9 G J .^{8}$ Table 5 provides an overview of the assumptions and results of these investigations.

Table 5: Assumptions and load estimates for unmitigated and mitigated disruptions in DEMO

\begin{tabular}{|l|l|l|}
\hline & $\begin{array}{l}\text { Unmitigated } \\
\text { disruption }\end{array}$ & $\begin{array}{l}\text { Mitigated } \\
\text { disruption }\end{array}$ \\
\hline $\begin{array}{l}\text { Thermal energy } \\
\text { content }\end{array}$ & $0.9 G J$ & $0.9 G J$ \\
\hline $\begin{array}{l}\text { Magnetics energy } \\
\text { content }\end{array}$ & $0.9 G J$ & $0.9 G J$ \\
\hline $\begin{array}{l}\text { Duration of wall } \\
\text { energy impact }\end{array}$ & $\begin{array}{l}\text { rise phase: 1ms, } \\
\text { decay phase: 3ms }\end{array}$ & 5 -10ms \\
\hline $\begin{array}{l}\text { Energy release } \\
\text { mechanism }\end{array}$ & $\begin{array}{l}\text { Conduction / } \\
\text { convection by } \\
\text { charged thermal } \\
\text { particles }\end{array}$ & Radiation \\
\hline Pre disruption $\lambda_{q}$ & 5 mm & not relevant \\
\hline $\begin{array}{l}\text { Radial broadening } \\
\text { factor }\end{array}$ & 3 & not relevant \\
\hline $\begin{array}{l}\text { Toroidal peaking } \\
\text { factor }\end{array}$ & 1 & 1.4 \\
\hline Peak energy density & $\begin{array}{l}\text { rise phase: } \\
3 M J / m^{2}, \text { decay } \\
\text { phase: } 7 M^{2} / m^{2}\end{array}$ & $0.75 M J / m^{2}$ \\
\hline $\begin{array}{l}\text { Peak energy impact } \\
\text { factor }\end{array}$ & $\begin{array}{l}95 M J /\left(m^{2} \sqrt{s}\right), \\
\text { decay phase: } \\
128 M J /\left(m^{2} \sqrt{s}\right)\end{array}$ & $\begin{array}{l}10.5- \\
7.5 M J /\left(m^{2} \sqrt{s}\right)\end{array}$ \\
\hline
\end{tabular}

For an unmitigated major disruption the heat load to the first wall via conduction/convection by charged thermal particles is calculated. We use an optimistic predisruption e-folding length of $5 \mathrm{~mm}^{9}$ in combination with a conservative value of 3 for the broadening of the radial deposition profile during the disruption []. Only loss of thermal energy is accounted for and it is assumed that $30 \%(70 \%)$ is lost in the rise phase (decay phase) lasting $1 \mathrm{~ms}(3 \mathrm{~ms})$ [38]. It is currently not clear, if there is toroidal peaking and we make here the optimistic assumption that this is not the case. Using a relatively simple tool that calculates in $2 \mathrm{D}$ the evolution of the power flux density evolution at the first wall leads to a peak energy flux density of $3 \mathrm{MJ} / \mathrm{m}^{2}\left(7 \mathrm{MJ} / \mathrm{m}^{2}\right)$ and peak heat impact factor of $95 M J /\left(m^{2} \sqrt{s}\right)\left(128 M J /\left(m^{2} \sqrt{s}\right)\right)$. This is far beyond the threshold for tile surface melting of $\mathrm{W}$ [7] and hence has to be absolutely avoided, as such an event is likely to necessitate an exchange of the affected blanket modules. The presented evaluation for unmitigated disruptions does not take into account any self-protecting mechanisms like vapor (or plasma) shielding, set up as a consequence of the sudden ablation of the plasma facing material surface at the beginning of the thermal quench.

The prediction of the wall load during a perfectly mitigated disruption is based on a simple model, in which the impurities injected by a disruption mitigation system are

\footnotetext{
${ }^{8}$ There is some deviation from the precise values of EU DEMO1 2015: $\beta_{\text {pol,tot }}=1.1$ and $E_{\text {therm }}=1.3 G J$

${ }^{9}$ This compares to a prediction of $\approx 1 \mathrm{~mm}$ in [13]
}

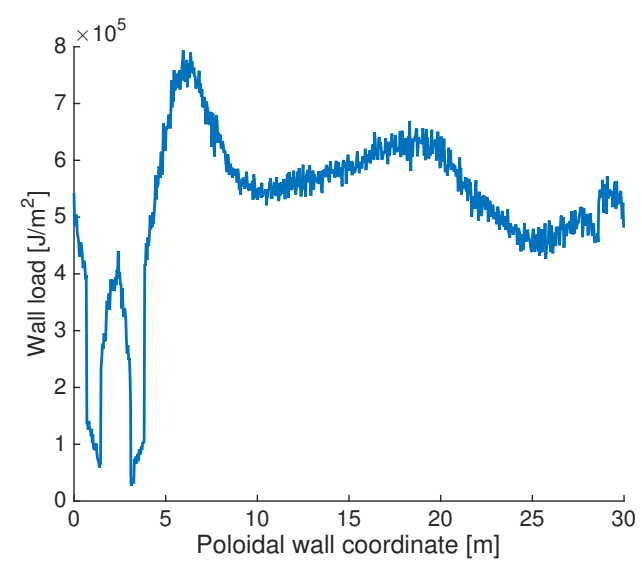

Figure 7: Poloidal distribution of the radiation wall load during a mitigated disruption in DEMO: The two minima roughly correspond to the divertor strike point areas

stopped at the edge of the plasma, resulting in a cold front moving inward until it crosses the $q=2$ surface and destabilizes MHD modes driving the thermal quench [39]. We assume that $100 \%$ of the thermal energy is radiated during these processes in $5-10 \mathrm{~ms}$ [40]. For the toroidal peaking factor the value 1.4 as observed at JET [41] is used, stressing that there are significant physical and technical uncertainties on this value. A Monte-Carlo-simulation tool similar to the one used for the calculation of static radiation loads [23], is used for estimating the first wall heat loads due radiation induced by massive gas injection. As a first approximation - following the simulation carried out for a basic inductive 15MA ITER scenario, with Ne massive gas injection before the thermal quench [42] - the plasma thermal energy was simulated to be radiated homogeneously in an annular region $(0.85 \leq r / a \leq 0.95)$ at the plasma edge. The distribution of energy density distributed to the first wall is shown in figure 7 . The peak energy flux density is $0.75 \mathrm{MJ} / \mathrm{m}^{2}$ and the peak heat impact factor is $10.5-7.5 \mathrm{MJ} /\left(\mathrm{m}^{2} \sqrt{s}\right)$.

\section{Particle loads}

The extent of the influx and the velocity distribution of particles hitting the wall defines the amount of eroded $\mathrm{W}$ at the first wall. In [43] initial estimates of the W sputtering rate are presented employing the code CELLSOR, which has been developed specifically to evaluate this issue in the context of system codes. CELLSOR consists of an analytical treatment of the plasma in the scrape-off layer (SOL) for fuel ions, solving the $1.5 \mathrm{~d}$ continuity equation in fluid approximation to obtain perpendicular flux and ion density in the SOL, and a fast Monto-Carlo description of the neutral particle $(\mathrm{D}, \mathrm{T})$ behavior. The trajectories of neutral $\mathrm{W}$ are computed within CELLSOR ERO, an add-on code used for calculations of prompt redeposition and self-sputtering. Within the analytic description of wall 
erosion by ions (i.e. by fuel, ash (He), seeding gas (N, Ar, $\mathrm{Kr}$ ) and wall material (W)) impurity concentrations are assumed to be radially constant.

For the core plasma, pre-scribed radial profiles of density and temperature are used, including a parametric model of the pedestal profiles. The primary erosion of the tungsten first wall is calculated based on contributions by the plasma fluxes (fuel and impurity ions), including the acceleration by a sheath in front of the wall, as well as by energetic $(>1 \mathrm{keV})$ neutrals which are mainly originating from charge exchange $(\mathrm{CX})$ collisions of the recycled neutrals from the wall re-entering the hot pedestal region. In this description, the primary erosion of the first wall depends on the radial transport coefficients (diffusivity, convection), the parallel time of transport to the divertor, the gap between separatrix and first wall and the plasma parameters in the pedestal (temperature at the top / separatrix).

For a systematic study 6 test cases were chosen (table 6 ) covering a reasonable range of SOL regimes from weak to strong diffusive transport up to a density shoulder formation case with strong convective transport. The intermediate case assumes $D_{\perp}=0.5 \mathrm{~m}^{2} / \mathrm{s}$ and $v_{\perp}=0.5 \mathrm{~m} / \mathrm{s}$. ???Review and comment???

Table 6: SOL physics cases distinguished by diffusive and convective radial transport strength and separatrix density.

\begin{tabular}{|l|l|l|l|l|}
\hline Test case & $D_{\perp}\left[\mathrm{m}^{2} / \mathrm{s}\right]$ & $v_{\perp}[\mathrm{m} / \mathrm{s}]$ & $\begin{array}{l}n_{s e p} \\
{\left[10^{20} \mathrm{~m}^{-3}\right]}\end{array}$ & $\begin{array}{l}\Gamma_{\perp}\left(\Delta_{S O L}\right. \\
0.1 \mathrm{~m}) \\
{\left[10^{20} /\left(\mathrm{m}^{2} \mathrm{~s}\right)\right.}\end{array}$ \\
\hline $\begin{array}{l}\text { Intermediate } \\
\text { case }\end{array}$ & 0.5 & 5 & 0.5 & 2.92 \\
\hline $\begin{array}{l}\text { Weak } \\
\text { transport }\end{array}$ & 0.1 & 0.1 & 0.5 & 0.07 \\
\hline $\begin{array}{l}\text { Strong } \\
\text { transport }\end{array}$ & 1 & 5 & 0.5 & 6.61 \\
\hline $\begin{array}{l}\text { Low } \\
\text { density }\end{array}$ & 0.5 & 5 & 0.25 & 1.36 \\
\hline $\begin{array}{l}\text { High } \\
\text { density }\end{array}$ & 0.5 & 5 & 1 & 6.75 \\
\hline $\begin{array}{l}\text { Density } \\
\text { shoulder }\end{array}$ & 0.5 & 25 & 0.5 & 24.09 \\
\hline
\end{tabular}

Figure 8 shows for these test cases the $\mathrm{W}$ erosion rate as a function of wall clearance $\Delta_{S O L}$. It is obvious that increasing $\Delta_{S O L}$ for all cases significantly reduces the erosion rate. The picture also shows a horizontal line corresponding to eroding the full W armor (assumed thickness: $2 \mathrm{~mm}$, total weight $54 \mathrm{t}$ ) within the life time of the starter blanket of 2 full power years (fpy). It can be seen that especially for the cases of the density shoulder a clearance of more than $0.3 \mathrm{~m}$ is necessary, while for the intermediate case $0.08 \mathrm{~m}$ would be sufficient.

In a next step poloidal resolution and conservation of energy should be implemented into the analysis code. The resulting value of $\Delta_{S O L}$ will be used as a boundary condition in the determination of the wall contour. Certainly it has to be investigated, if the necessary decrease of the elongation in order to maintain vertical stability is acceptable in terms of performance.

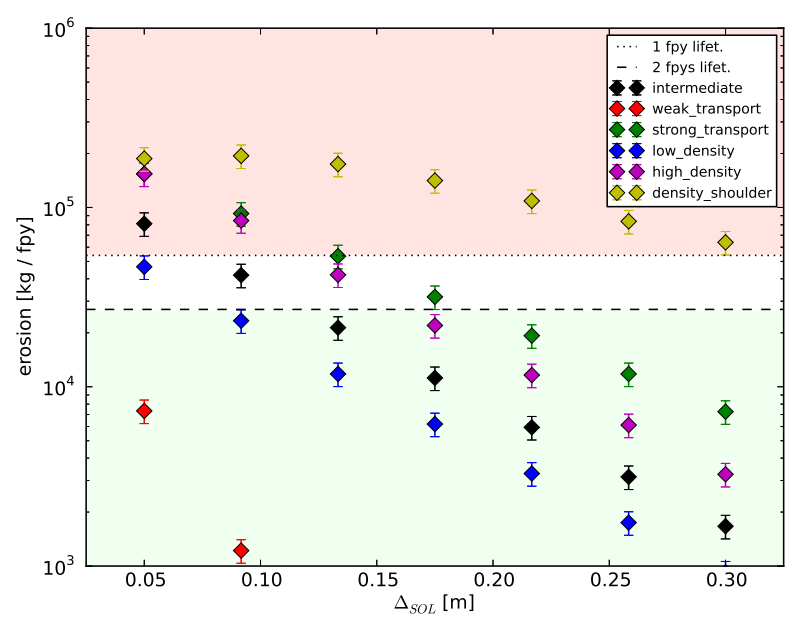

Figure 8: Net mass erosion in kg / fpy by ions (D, T, He, N,W) and neutrals $(\mathrm{D}, \mathrm{T})$ as a function of wall clearance $\Delta_{S O L}$ for the SOL test cases described in table 6

\section{Summary}

This publication summarizes the recent knowledge and open gaps with respect to the question of first wall loads in DEMO, with a focus on static wall loads. In section 2 various technical load limits are introduced. One of the most constraining aspects for the case of static loads is the limit of $550^{\circ} \mathrm{C}$ for EUROFER-97. An initial list of load types and sets of conservative assumptions on the input power distribution are presented in section 3 .

The characteristics of the blob transport determines the distribution of heat transported by charged particles to the first wall. In principle by increasing the wall clearance it is possible to shift this balance as much as necessary towards the divertor. However, the higher the fraction of heat transported in blobs and the associated e-folding length are, the higher the clearance needs to be and the lower will be the vertically stabilizing effect of the vessel and the lower the elongation and hence the performance of the device can be.

For the integrated optimization of plasma shape and first wall engineering design a framework including 3D heat load analysis has been established. Employing an initial engineering wall design with clear optimization potential in combination with parameters for the flat-top phase (x-point configuration), loads up to $7 \mathrm{MW} / \mathrm{m}^{2}$ have been calculated. Similar evaluations for limited phases have to follow. As shown for the ITER case [20], a critical point will be the question of identifying appropriate penalties (ITER: in total up to 2.44) representing all relevant kinds of inaccuracies to be multiplied by the peak heat loads. Increasing the level of radiation by impurity seeding is used as a method to homogenize the heat distribution at the first wall. The radiation source distribution in the poloidal plane is an uncertainty in this context. Assuming a fraction of power radiated from the $\mathrm{x}$-point region 
between $1 / 5$ and $1 / 3$ leads to peaks of the total power flux density due to radiation of $0.6-0.8 \mathrm{MW} / \mathrm{m}^{2}$, found in the outer baffle region.

Investigations of fast particle heat loads on the first wall with orbit following codes suggest, that even taking into account poloidal and toroidal peaking, the highest loads due not exceed $0.1 \mathrm{MW} / \mathrm{m}^{2}$. In a linked investigation it has been shown, that the effect of the plasma response is negligible in this case. Effects of the interaction of modes and fast particles have not been taken into account, but might significantly change the result.

An investigation of the heat impact and its dynamics for unmitigated disruptions shows very clear that it must be an absolute priority to avoid these events in DEMO. The calculated heat impact factor for mitigated disruptions is about a factor of 2 below the surface melt threshold for $\mathrm{W}$. However the ITER experience shows, that redoing such an analysis with an engineering design of the first wall and a more detailed description of the plasma often leads to a significant increase of the calculated loads.

????Particle loads?????

From this initial findings on first wall loads in DEMO some early recommendations for the design of the first wall can be derived. Two fundamental options for the first wall design are discussed: (1) An ITER-shaped wall that is within the limits of the breeding blanket manufacturing possibilities aligned to the flux surfaces and does not contain any high heat flux components and (2) a wall that contains high heat flux limiters elongated in the poloidal direction. Also a mixture of these two options is conceivable. Furthermore, a double-null configuration for DEMO is discussed and will be subject of future publications. As it is assumed at the moment that the risk of unmitigated disruptions cannot be reduced sufficiently, the implementation of sacrificial limiters that receive the heat impact during these events is advisable. Especially for the outer baffle region, which is heavily statically loaded by charged particles and radiation, a solution with higher heat flux capability might become necessary. After a detailed study of dynamic events (e.g. vertical displacement events and unforeseen H-L-transitions) more locations that need to be hardened against higher heat fluxes than $1 M W / m^{2}$ could be identified.

\section{Acknowledgment}

This work has been carried out within the framework of the EUROfusion Consortium and has received funding from the European Union's Horizon 2020 research and innovation programme under grant agreement number 633053. The views and opinions expressed herein do not necessarily reflect those of the European Commission, nor of the ITER organization..

[1] R. Wenninger et al. Demo exhaust challenges beyond iter. 42nd
EPS Conference on Controlled Fusion and Plasma Physics, 2015.

[2] A.René Raffray and Gianfranco Federici. Raclette: a model for evaluating the thermal response of plasma facing components to slow high power plasma transients. part i: Theory and description of model capabilities. Journal of Nuclear Materials, 244(2):85 - 100, 1997.

[3] R. Mitteau, P. Stangeby, C. Lowry, M. Firdaouss, H. Labidi, A. Loarte, M. Merola, R. Pitts, and R. Raffray. A shaped first wall for \{ITER\}. Journal of Nuclear Materials, 415(1, Supplement):S969 - S972, 2011. Proceedings of the 19th International Conference on Plasma-Surface Interactions in Controlled Fusion.

[4] G. Federici et al. European demo design strategy and consequences for materials. European DEMO design strategy and consequences for materials, 2016.

[5] Meimei Li, M.A. Sokolov, and S.J. Zinkle. Tensile and fracture toughness properties of neutron-irradiated cucrzr. Journal of Nuclear Materials, 393(1):36 - 46, 2009.

[6] T. Hirai, F. Escourbiac, S. Carpentier-Chouchana, A. Fedosov, L. Ferrand, T. Jokinen, V. Komarov, A. Kukushkin, M. Merola, R. Mitteau, R.A. Pitts, W. Shu, M. Sugihara, B. Riccardi, S. Suzuki, and R. Villari. \{ITER\} tungsten divertor design development and qualification program. Fusion Engineering and Design, 88(9-10):1798 - 1801, 2013.

[7] Materials for nuclear energy systems. World Academy of Ceramics? FORUM.

[8] F. Maviglia et al. Contribution to SOFT conference 2016, 2016.

[9] Y R Martin, T Takizuka, and the ITPA CDBM H-mode Threshold Database Working Group. Power requirement for accessing the h-mode in iter. Journal of Physics: Conference Series, 123(1):012033, 2008.

[10] F. Ryter, S.K. Rathgeber, L. Barrera Orte, M. Bernert, G.D. Conway, R. Fischer, T. Happel, B. Kurzan, R.M. McDermott, A. Scarabosio, W. Suttrop, E. Viezzer, M. Willensdorfer, E. Wolfrum, and the ASDEX Upgrade Team. Survey of the $\mathrm{h}$-mode power threshold and transition physics studies in asdex upgrade. Nuclear Fusion, 53(11):113003, 2013.

[11] R.J. Goldston. Heuristic drift-based model of the power scrapeoff width in low-gas-puff h-mode tokamaks. Nuclear Fusion, 52(1):013009, 2012.

[12] T. Eich, B. Sieglin, A. Scarabosio, W. Fundamenski, R. J. Goldston, and A. Herrmann. Inter-elm power decay length for jet and asdex upgrade: Measurement and comparison with heuristic drift-based model. Phys. Rev. Lett., 107:215001, 2011.

[13] R.P. Wenninger, M. Bernert, T. Eich, E. Fable, G. Federici, A. Kallenbach, A. Loarte, C. Lowry, D. McDonald, R. Neu, T. Pütterich, P. Schneider, B. Sieglin, G. Strohmayer, F. Reimold, and M. Wischmeier. Demo divertor limitations during and in between elms. Nuclear Fusion, 54(11):114003, 2014.

[14] J. R. Myra, D. A. Russell, and D. A. D'Ippolito. Collisionality and magnetic geometry effects on tokamak edge turbulent transport. i. a two-region model with application to blobs. Physics of Plasmas, 13(11), 2006.

[15] D. Carralero, P. Manz, L. Aho-Mantila, G. Birkenmeier, M. Brix, M. Groth, H. W. Müller, U. Stroth, N. Vianello, and E. Wolfrum. Experimental validation of a filament transport model in turbulent magnetized plasmas. Phys. Rev. Lett., 115:215002, Nov 2015.

[16] M. Siccinio et al. Analytical models for the evaluation of thermal loads on divertor and first wall in demo. PSI conference, 2016.

[17] H.W. Müller, M. Bernert, D. Carralero, A. Kallenbach, B. Kurzan, A. Scarabosio, B. Sieglin, L. Tophøj, N. Vianello, and E. Wolfrum. Far scrape-off layer particle and heat fluxes in high density - high power scenarios. Journal of $\mathrm{Nu}$ clear Materials, 463:739 - 743, 2015. PLASMA-SURFACE \{INTERACTIONS\} 21Proceedings of the 21st International Conference on Plasma-Surface Interactions in Controlled Fusion Devices Kanazawa, Japan May 26-30, 2014.

[18] M Bernert, T Eich, A Kallenbach, D Carralero, A Huber, 
P T Lang, S Potzel, F Reimold, J Schweinzer, E Viezzer, and $\mathrm{H}$ Zohm. The h-mode density limit in the full tungsten asdex upgrade tokamak. Plasma Physics and Controlled Fusion, 57(1):014038, 2015.

[19] G. Birkenmeier, P. Manz, D. Carralero, F.M. Laggner, G. Fuchert, K. Krieger, H. Maier, F. Reimold, K. Schmid, R. Dux, T. Pütterich, M. Willensdorfer, E. Wolfrum, and The ASDEX Upgrade Team. Filament transport, warm ions and erosion in asdex upgrade l-modes. Nuclear Fusion, 55(3):033018, 2015.

[20] R. Mitteau, P. Stangeby, H. Labidi, R. Bruno, and R. Raffray. The combined effects of magnetic asymmetry, assembly and manufacturing tolerances on the plasma heat load to the \{ITER\} first wall. Journal of Nuclear Materials, 463:411 - 414, 2015. PLASMA-SURFACE \{INTERACTIONS\} 21Proceedings of the 21st International Conference on Plasma-Surface Interactions in Controlled Fusion Devices Kanazawa, Japan May 26-30, 2014

[21] H. Zohm. Internal report, 2015.

[22] M. Firdaouss, V. Riccardo, V. Martin, G. Arnoux, and C. Reux. Modelling of power deposition on the $\{$ JET $\}$ ITER $\}$ like wall using the code \{PFCFLux\}. Journal of Nuclear Materials, 438, Supplement:S536 - S539, 2013. Proceedings of the 20th International Conference on Plasma-Surface Interactions in Controlled Fusion Devices

[23] R. Wenninger, F. Arbeiter, J. Aubert, L. Aho-Mantila, R. Albanese, R. Ambrosino, C. Angioni, J.-F. Artaud, M. Bernert, E. Fable, A. Fasoli, G. Federici, J. Garcia, G. Giruzzi, F. Jenko, P. Maget, M. Mattei, F. Maviglia, E. Poli, G. Ramogida, C. Reux, M. Schneider, B. Sieglin, F. Villone, M. Wischmeier, and H. Zohm. Advances in the physics basis for the european demo design. Nuclear Fusion, 55(6):063003, 2015.

[24] The physics and technology basis entering european system code studies for demo", author=,wenninger, r. and others. Nuclear Fusion, submitted, 2016.

[25] J Horacek, R A Pitts, J Adamek, G Arnoux, J-G Bak, S Brezinsek, M Dimitrova, R J Goldston, J P Gunn, J Havlicek, SH Hong, F Janky, B LaBombard, S Marsen, G Maddaluno, L Nie, V Pericoli, Tsv Popov, R Panek, D Rudakov, J Seidl, D S Seo, M Shimada, C Silva, P C Stangeby, B Viola, P Vondracek, H Wang, G S Xu, Y Xu, and JET Contributors. Multimachine scaling of the main sol parallel heat flux width in tokamak limiter plasmas. Plasma Physics and Controlled Fusion, 58(7):074005, 2016.

[26] M. Kocan, R.A. Pitts, G. Arnoux, I. Balboa, P.C. de Vries, R. Dejarnac, I. Furno, R.J. Goldston, Y. Gribov, J. Horacek, M. Komm, B. Labit, B. LaBombard, C.J. Lasnier, R. Mitteau, F. Nespoli, D. Pace, R. Panek, P.C. Stangeby, J.L. Terry, C. Tsui, and P. Vondracek. Impact of a narrow limiter sol heat flux channel on the iter first wall panel shaping. Nuclear Fusion, 55(3):033019, 2015

[27] C. Silva, G. Arnoux, S. Devaux, D. Frigione, M. Groth, J. Horacek, P.J. Lomas, S. Marsen, G. Matthews, L. Meneses, R.A. Pitts, and JET-EFDA Contributors. Characterization of scrape-off layer transport in jet limiter plasmas. Nuclear Fusion, 54(8):083022, 2014.

[28] R. Dux. Lab Report, Max-Planck-Institut fuer Plasmaphysik, $10 / 30,2006$

[29] G. V. Pereverzev et al. Lab Report, Max-Planck-Institut fuer Plasmaphysik, 5/42, 1991.

[30] E Fable, C Angioni, F J Casson, D Told, A A Ivanov, F Jenko, R M McDermott, S Yu Medvedev, G V Pereverzev, F Ryter, W Treutterer, E Viezzer, and the ASDEX Upgrade Team. Novel free-boundary equilibrium and transport solver with theorybased models and its validation against asdex upgrade current ramp scenarios. Plasma Physics and Controlled Fusion, 55(12):124028, 2013.

[31] M. Bernert et al. 42nd EPS Conference on Controlled Fusion and Plasma Physics, 2015.

[32] A. Huber et al. Impact of strong impurity seeding on the radiation losses in jet with iter-like wall. 41st EPS Conference on
Controlled Fusion and Plasma Physics, 2014.

[33] M. Bernert et al. PSI conference, 2016.

[34] E. Hirvijoki, O. Asunta, T. Koskela, T. Kurki-Suonio, J. Miettunen, S. Sipilä, A. Snicker, and S. Äkäslompolo. Ascot: Solving the kinetic equation of minority particle species in tokamak plasmas. Computer Physics Communications, 185(4):1310 - 1321, 2014.

[35] TD. Pfefferlé et al. Nuclear Fusion - accepted, 2016.

[36] David Pfefferlé, Cyril Misev, Wilfred A. Cooper, and Jonathan P. Graves. Impact of rmp magnetic field simulation models on fast ion losses. Nuclear Fusion, 55(1):012001, 2015.

[37] D. Pfefferlé, W.A. Cooper, J.P. Graves, and C. Misev. Venuslevis and its spline-fourier interpolation of $3 \mathrm{~d}$ toroidal magnetic field representation for guiding-centre and full-orbit simulations of charged energetic particles. Computer Physics Communications, 185(12):3127 - 3140, 2014.

[38] G. Pautasso et al. Details of the power deposition in the thermal quench of asdex upgrade disruptions. EPS Conference on Controlled Fusion, 2004

[39] A. Fil et al. Modelling of disruption mitigation by massive gas injection. 41st EPS Conference on Controlled Fusion and Plasma Physics, 2014.

[40] Plasma facing materials performance under iter-relevant mitigated disruption photonic heat loads. Journal of $\mathrm{Nu}$ clear Materials, 463:61 - 65, 2015. PLASMA-SURFACE \{INTERACTIONS\} 21Proceedings of the 21st International Conference on Plasma-Surface Interactions in Controlled Fusion Devices Kanazawa, Japan May 26-30, 2014.

[41] P. Drewelow et al. Measurement of radiation asymmetries during disruption mitigation at jet. 42nd EPS Conference on Controlled Fusion and Plasma Physics, 42, 2015.

[42] V. Levnov et al. Modeling of pre-thermal quench stage of disruption in iter triggered by massive gas injection. 42nd EPS Conference on Controlled Fusion and Plasma Physics, 42, 2015.

[43] M. Beckers et al. PSI conference, 2016. 